QUARTERLY OF APPLIED MATHEMATICS

VOLUME LXIV, NUMBER 2

JUNE 2006, PAGES 321-333

S $0033-569 X(06) 01023-7$

Article electronically published on May 3, 2006

\title{
ON LOCAL UNIQUENESS IN NONLINEAR ELASTODYNAMICS
}

\author{
$\mathrm{BY}$ \\ R. J. KNOPS \\ School of Mathematical and Computer Sciences, Heriot-Watt University, Edinburgh EH14 $4 A S$, \\ Scotland, U.K.
}

\begin{abstract}
A conservation law, derived from properties of the energy-momentum tensor, is used to establish uniqueness of suitably constrained solutions to the initial boundary value problem of nonlinear elastodynamics. It is assumed that the region is star-shaped, that the data are affine, and that the strain-energy function is strictly rank-one convex and quasi-convex. It is shown how these assumptions may be successively relaxed provided that the class of considered solutions is correspondingly further constrained.
\end{abstract}

1. Introduction. This paper examines uniqueness of locally smooth solutions to the initial displacement boundary value problem of nonlinear homogeneous elastodynamics in the absence of body-forces and on a bounded three-dimensional region. Weak solutions are not necessarily unique, and smooth solutions do not in general exist globally in time. Consequently, uniqueness is sought only in the class of sufficiently smooth solutions on the maximal time interval of existence. Other investigations of uniqueness pertinent to this problem include those by Hughes, Kato and Marsden [15, Wheeler [26], and Dafermos [7], 8] who all assume a strongly elliptic strain energy function. In particular, 15. demonstrates that in $\mathbb{R}^{n}$ a unique solution exists locally of class $H^{(s+1)} \times H^{s}$ for initial data in the same class, where $H^{s}$ is the usual Hilbert space of order $s$, and $s>n / 2+1$. The discussion in [7], that extends to thermoelastodynamics, considers a strongly elliptic entropy function and from estimates for continuous dependence on initial data obtains uniqueness of a smooth solution in the class of weak solutions. A similar result is established in [8] for a poly-convex entropy subject to certain other conditions. A different convexity assumption, further examined by Sofer [25], is adopted by Knops, Levine and Payne [20, who likewise establish uniqueness as a consequence of continuous dependence estimates.

Received July 25, 2005.

2000 Mathematics Subject Classification. Primary 74B20; Secondary 74H25.

Key words and phrases. Nonlinear elastodynamics, uniqueness, constrained solutions.

(C)2006 Brown University 
By constrast, the present study considers a strain energy function that, although eventually required merely to be bounded, initially is assumed to be quasi-convex and (strictly) rank-one convex. The corresponding data must then be affine, the region star-shaped, and deformation and velocity gradients together with stress suitably constrained. The generalised notions of convexity and restriction to a star-shaped region are introduced by Knops and Stuart 21] in their discussion of uniqueness for the affine displacement boundary value problem of nonlinear homogeneous elasticity without body force. Similar to 21, a crucial component of the proof presented here is a conservation law, or integral identity, derived from properties of the energy-momentum (Eshelby) tensor which now, however, is used to construct a differential inequality leading to a contradiction and consequent uniqueness.

The generalised convexity notions, affine data, and star-shaped region are successively discarded in favour of the deformation being further constrained by bounds on the strain energy and additional bounds on higher order deformation gradients. The final result involves no convexity assumptions and is consistent with the well-known conclusion for the initial boundary value problem of linear elastodynamics where only existence of a strain energy is required for uniqueness. (See Brun [4, [5] or Knops and Payne [1], [19]). The absence of generalised convexity conditions, even for smooth data, is a likely cause of instability, but it is worth remarking that the imposition of constraints is well known in the stabilisation of ill-posed problems.

Section 2 disposes of notation, defines the initial displacement boundary value problem, and derives the conservation law subsequently required. Requisite properties of the difference between two distinct locally smooth solutions are also established. Further average properties of this difference in terms of a certain time dependent function are presented in Section 3, and a differential inequality constructed for this function. Section 4 uses the inequality together with a quasi-convex and rank-one convex strain energy function to demonstrate uniqueness of a suitably constrained solution to the affine displacement boundary value problem on a star-shaped region. The constraints prescribe bounds on the deformation and velocity gradients, and also on the PiolaKirchhoff stress tensor. Finally, Section 5 first discards the notion of quasi-convexity to obtain uniqueness for general but sufficiently smooth initial and displacement boundary data in the class of locally smooth solutions further constrained by additional prescribed bounds on third order deformation gradients, and the strain energy. Rank-one convexity and a star-shaped region are subsequently also abandoned, and uniqueness established for locally smooth solutions which, besides the constraints already introduced, satisfy certain bounded boundary behaviour. All the uniqueness proofs depend upon a contradiction obtained from the same fundamental differential inequality adapted to the various constitutive assumptions and constraints on the solution. The respective treatments, however, require only slight modification to accommodate the different circumstances.

It is assumed throughout that the data are compatible with the existence of an appropriately smooth solution on a maximal time interval.

A preliminary version of the results reported here is included in [17]. 
2. Notation and basic concepts. A nonlinear homogeneous compressible elastic material in its reference configuration occupies the bounded region $\Omega \in \mathbb{R}^{3}$ of threedimensional Euclidean space and has piecewise smooth boundary $\partial \Omega$ on which the unit outward normal is $N$. The material is set in motion by prescribed initial defomation and velocity, together with specified time-dependent boundary deformation. Zero body force is assumed. With respect to a given Cartesian coordinate system, the point in $\Omega$ whose vector position is $X$ deforms into the point whose position at time $t$ is $x=(X, t)$.

A direct notation is mainly employed in which, for example, the tensor gradient operator with respect to the variable $X$ is denoted by $\nabla$ so that the components of the deformation gradient $F$ are $F_{i j}=(\nabla x)_{i j}=\partial x_{i} / \partial X_{j}$, where $X_{i}, x_{i}, i=1,2,3$, are the cartesian components of the vectors $X, x$, respectively. The corresponding tensor divergence operator is denoted by Div, while the tensor product of vectors $a, b$ is indicated by $a \otimes b$, transposition by superscript $T$, and the unit tensor by $I$. There is no typographical distinction between scalar, vector and tensor quantities, whose meaning should be clear from the context. The inner product of vectors and tensors is denoted by $A B=\operatorname{tr} A B^{T}$, where $t r$ is the trace operator. The set of deformation gradients having positive determinant is denoted by $\mathrm{M}^{+}$, and a subscript comma indicates partial differentiation with respect to the relevant variable.

The elastic material possesses a strain energy function $W(F) \in C^{1}\left(M^{+}, \mathbb{R}\right)$ per unit volume of $\Omega$ that is dependent only upon the deformation gradient $F$ in view of the assumed material homogeneity. The Piola-Kirchhoff stress tensor $T$ is related to the strain energy function by

$$
T(F)=\frac{\partial W}{\partial F}(F) .
$$

For the initial boundary value problem considered here, it is supposed that sufficiently smooth solutions $x(X, t)$ exist locally on some finite maximal time interval which by appropriate rescaling of the time variable may be taken to be $[0,1]$. In the absence of body-forces the governing equations of motion satisfied by the smooth solution are

$$
\operatorname{Div} T(F)=\rho \ddot{x}, \quad(X, t) \in \Omega \times[0,1]
$$

where $\rho$ is the suitably rescaled uniform mass density in the reference configuration and a superposed dot indicates material time differentiation. The corresponding initial and boundary conditions are given by

$$
x(X, 0)=u(X), \quad \dot{x}(X, 0)=v(X), \quad X \in \Omega,
$$

and

$$
x(X, t)=g(X, t), \quad(X, t) \in \partial \Omega \times[0,1],
$$

for prescribed vector functions $u, v, g$.

Because it is assumed that $x$ is sufficiently smooth, (2.2) may be multiplied by $\dot{x}$ and the resulting equation integrated over $\Omega \times[0,1]$ to obtain the balance of energy in the 
form

$$
\begin{aligned}
\int_{\Omega(t)}\left[W(\nabla x)+\frac{\rho}{2} \dot{x} \dot{x}\right] d X= & \int_{0}^{t} \oint_{\partial \Omega(\eta)} \operatorname{tr} T(\nabla x) \dot{x} \otimes X d X d \eta \\
& +\int_{\Omega(0)}\left[W(\nabla u)+\frac{\rho}{2} v v\right] d X
\end{aligned}
$$

where here and throughout, the subscript $\Omega(t)$, for example, attached to an integral signifies that the corresponding integrand is to be evaluated at time $t$.

The subsequent uniqueness proof depends in part upon an integral identity or conservation law which may be derived from relevant properties of the energy-momentum (Eshelby) tensor defined by

$$
\mathbb{B}=F^{T} T-\left(W-\frac{1}{2} \rho \dot{x} \dot{x}\right) I .
$$

Multiplication of (2.2) by $F$ leads to the relation

$$
\operatorname{Div} \mathbb{B}=\rho\left(F^{T} \dot{x}\right), \quad(X, t) \in \Omega \times[0,1],
$$

which, after further multipication by $X$, implies the identity

$$
\operatorname{Div}\left(X \mathbb{B}^{T}\right)-\operatorname{tr} \mathbb{B}=\rho X\left(F^{T} \dot{x}\right)^{\prime}, \quad(X, t) \in \Omega \times[0,1] .
$$

Integration over $\Omega \times[0,1]$, substitution for $\mathbb{B}$ from (2.6) and subsequent rearrangement then yields for $t \in[0,1]$

$$
\begin{aligned}
\int_{0}^{t} & \int_{\Omega(\eta)}\left[6 W(F)+5 \rho x x_{, \eta \eta}\right] d X d \eta \\
\quad & =3\left[\int_{\Omega(t)} \rho x \dot{x} d X-\int_{\Omega(0)} \rho u v d X\right]+2\left[\int_{\Omega(t)} \rho X F^{T} \dot{x} d X-\int_{\Omega(0)} \rho X F^{T} \dot{x} d X\right] \\
& +2 \int_{0}^{t} \int_{\partial \Omega(\eta)}\left[\operatorname{tr} T N \otimes(x-X G r a d x)+N X\left(W(F)-\frac{1}{2} \rho x_{, \eta} x_{, \eta}\right)\right] d S d \eta .
\end{aligned}
$$

Identity (2.9), previously derived by Fletcher 11 for linear elastodynamics, is the counterpart in elastodynamics of the conservation law which in elastostatics has been discussed by several authors including Chadwick [6], Eshelby [10, Green [13, Hill [14, and Knowles and Sternberg [22. Appropriate weak formulations are considered by Gelfand and Fomin [12] (see also Ericksen [9] and Maugin [23]) using the variational calculus. The elastostatic conservation law is employed by Knops and Stuart 21] to establish uniqueness in the affine boundary value problem for nonlinear homogeneous elasticity on a bounded star-shaped region.

Now let $y(X, t)$ be a second smooth solution that satisfies (2.2) subject to the initial and boundary conditions (2.3) and (2.4). Assume without loss of generality that the maximal existence interval for $y$ contains the unit interval $[0,1]$, and put

$$
w(X, t)=x(X, t)-y(X, t), \quad(X, t) \in \bar{\Omega} \times[0,1],
$$


where a superposed bar denotes closure. It easily follows that the smooth function $w$ satisfies

$$
\begin{array}{rlrl}
\operatorname{Div} T(\nabla x)-\operatorname{Div} T(\nabla y) & =\rho \ddot{w}, & (X, t) \in \Omega \times[0,1], \\
w(X, 0)=\dot{w}(X, 0) & =0, \quad X \in \Omega, \\
w(X, t) & =0, \quad(X, t) \in \partial \Omega \times[0,1] .
\end{array}
$$

Identities satisfied by the function $w$ required later are stated and proved in the next lemma.

Lemma 2.1. The function $w$ defined in (2.10) satisfies for $t \in[0,1]$ the identities

$$
\begin{aligned}
\int_{\Omega(t)}[W(\nabla x)-W & \left.(\nabla y)+\frac{1}{2} \rho \dot{w} \dot{w}\right] d X \\
& =-\int_{0}^{t} \int_{\Omega(\eta)}\left[\rho w_{, \eta} y_{, \eta \eta}+\operatorname{tr}\{T(\nabla x)-T(\nabla y)\} \nabla y_{, \eta}\right] d X d \eta
\end{aligned}
$$

and

$$
\begin{aligned}
5 \int_{0}^{t} & \int_{\Omega(\eta)} \rho w w_{, \eta \eta} d X d \eta=3 \int_{\Omega(t)} \rho w \dot{w} d X+2 \int_{\Omega(t)} \rho \operatorname{tr} \nabla w X \otimes \dot{w} d X \\
& -6 \int_{0}^{t} \int_{\Omega(\eta)} \rho w y, \eta \eta \\
& -2 \int_{0}^{t} \int_{\Omega(\eta)} \rho t r \nabla y_{, \eta \eta} w \otimes X d X \int_{0}^{t} \int_{\Omega(\eta)} \operatorname{tr}[T(\nabla x)-T(\nabla y)][(X \nabla) \nabla y] d X d \eta
\end{aligned}
$$

where

$$
\begin{gathered}
G_{1}(t)=\int_{0}^{t} \int_{\Omega(\eta)}[W(\nabla x)-W(\nabla y)] d X d \eta, \\
G_{2}(t)=\int_{0}^{t} \int_{\partial \Omega(\eta)}\{N X[W(\nabla x)-W(\nabla y)]-\operatorname{tr} T(\nabla x)(X \nabla w) \otimes N\} d S d \eta .
\end{gathered}
$$

Proof. Identity (2.14) is established by subtraction of the respective energy balances (2.5) for the solutions $x$ and $y$, followed by an integration by parts and the use of (2.11) $-(2.13)$.

The second identity (2.15) is obtained by subtraction of the respective integral identities (2.9) for $x$ and $y$.

3. Further properties. Here we introduce the function $H(t)$ in terms of which the proofs of uniqueness are developed, and discuss relevant properties.

Definition 3.1. The function $H(t)$ for $t \in[0,1]$ is given by

$$
H(t)=\int_{0}^{t} \int_{\Omega(\eta)} \rho w w d X d \eta+\beta\left(t+t_{0}\right)^{2},
$$

where $\beta, t_{0}$ are positive constants to be determined, and $w(t)$ is given by (2.10). 
It may easily be proved that the following equivalent representations hold for the first and second derivatives of $H(t)$ :

$$
\begin{aligned}
\dot{H}(t) & =\int_{\Omega(t)} \rho w w d X+2 \beta\left(t+t_{0}\right) \\
& =2 \int_{0}^{t} \int_{\Omega(\eta)} \rho w w_{, \eta} d X d \eta+2 \beta\left(t+t_{0}\right), \\
\ddot{H}(t) & =2 \int_{\Omega(t)} \rho w \dot{w} d X+2 \beta \\
& =2 \int_{0}^{t} \int_{\Omega(\eta)} \rho\left[w_{, \eta} w_{, \eta}+w w_{, \eta \eta}\right] d X d \eta+2 \beta .
\end{aligned}
$$

An alternative expression to (3.5) for $\ddot{H}(t)$, obtained from the identities presented in Lemma 2.1, is given by

$$
\begin{aligned}
\ddot{H}(t)= & 4 \int_{0}^{t} \int_{\Omega(\eta)} \rho w_{, \eta} w_{, \eta} d X d \eta+2 \int_{\Omega(t)} \rho \operatorname{tr} \nabla w \dot{w} \otimes X d X \\
& -8 G_{1}(t)+2 G_{2}(t)-2 G_{3}(t)+2 G_{4}(t)+2 \beta, \quad t \in[0,1],
\end{aligned}
$$

where $G_{1}(t)$ and $G_{2}(t)$ are defined in (2.16) and (2.17), and

$$
\begin{array}{r}
G_{3}(t)=\int_{0}^{t} \int_{\Omega(\eta)} \rho\left[(t-\eta) y_{, \eta \eta} w_{, \eta}+4 y_{, \eta \eta} w+(X \nabla) y_{, \eta \eta} w\right] d X d \eta, \\
G_{4}(t)=\int_{0}^{t} \int_{\Omega(\eta)} \operatorname{tr}\left[(t-\eta) \nabla y_{, \eta}-(X \nabla) \nabla y\right][T(\nabla x)-T(\nabla y)] d X d \eta,
\end{array}
$$

for $t \in[0,1]$.

We next prove that the function $H(t)$ satisfies a second order differential inequality.

Proposition 3.2 (Differential inequality). Let $\alpha>1$ be an otherwise arbitrary constant. The function $H(t)$ for $t \in[0,1]$ satisfies the differential inequality

$$
\begin{gathered}
H(t) \ddot{H}(t)+(\alpha-1) \dot{H}(t)^{2} \leq H(t)\left[2 \beta(2 \alpha-1)+2 \int_{\Omega(t)} \rho t r \nabla w \dot{w} \otimes X d X\right. \\
\left.-8(1+\alpha) G_{1}(t)+2 G_{2}(t)-2 G_{5}(t)+2 G_{6}(t)\right],
\end{gathered}
$$

where $G_{1}(t)$ and $G_{2}(t)$ are defined by (2.16) and (2.17), and

$$
\begin{gathered}
G_{5}(t)=4 \alpha \int_{0}^{t} \int_{\Omega(\eta)} \rho(t-\eta) y_{, \eta \eta} w_{, \eta} d X d \eta+G_{3}(t), \\
G_{6}(t)=4 \alpha \int_{0}^{t} \int_{\Omega(\eta)}(t-\eta) \operatorname{tr} \nabla y_{, \eta}[T(\nabla x)-T(\nabla y)] d X d \eta+G_{4}(t),
\end{gathered}
$$

with $G_{3}$ and $G_{4}$ given by (3.7) and (3.8).

Proof. In the identity

$$
\dot{H}^{2}(t)=4\left[\left\{\int_{0}^{t} \int_{\Omega(\eta)} \rho w w d X d \eta+\beta\left(t+t_{0}\right)^{2}\right\}\left\{\int_{0}^{t} \int_{\Omega(\eta)} \rho w_{, \eta} w_{, \eta} d X d \eta+\beta\right\}-S^{2}(t)\right],
$$


where

$$
\begin{aligned}
S^{2}(t)= & {\left[\int_{0}^{t} \int_{\Omega(\eta)} \rho w w d X d \eta+\beta\left(t+t_{0}\right)^{2}\right]\left[\int_{0}^{t} \int_{\Omega(\eta)} \rho w_{, \eta} w_{, \eta} d X d \eta+\beta\right] } \\
& -\left[\int_{0}^{t} \int_{\Omega(\eta)} \rho w w_{, \eta} d X d \eta+\beta\left(t+t_{0}\right)\right]^{2}
\end{aligned}
$$

we have by Schwarz's inequality that $S^{2} \geq 0$, and therefore after noting (3.1) we are led to

$$
H(t) \ddot{H}(t)+(\alpha-1) \dot{H}^{2}(t) \leq H(t) \ddot{H}(t)+4(\alpha-1) H(t)\left\{\int_{0}^{t} \int_{\Omega(\eta)} \rho w_{, \eta} w_{, \eta} d X d \eta+\beta\right\},
$$

since $\alpha>1$, and where $t \in[0,1]$. On the right side of (3.14) we replace $\ddot{H}(t)$ by its expression (3.6) ) and use the energy balance (2.14) to obtain (3.9).

This completes the assembly of preliminary material, and we now proceed to the discussion of uniqueness.

4. Uniqueness in the affine problem. In this section, we suppose that the strainenergy function $W$ satisfies generalised convexity conditions, and we consider uniqueness for the affine initial displacement boundary value problem on a star-shaped bounded region $\Omega$ for solutions in a stipulated class of bounded smooth functions on $\Omega \times[0,1]$. The affine data, convexity and star-shaped conditions are successively relaxed in the next section.

We include for completeness definitions of the generalised convexity conditions that the strain-energy function $W$ is assumed to satisfy. Comprehensive discussions of these and related concepts are presented in, for example, [1, 2, [3], and 24].

Definition 4.1 (Quasi-convexity). The function $W \in C\left(M^{+}, \mathbb{R}\right)$ is quasi-convex at (constant) $A \in M^{+}$if and only if

$$
\int_{D} W(A+\nabla \xi(X)) d X \geq \int_{D} W(A) d X
$$

for all nonempty open bounded subsets $D \subseteq \mathbb{R}^{3}$ and all Lipschitz continuous functions $\xi$ that vanish on $\partial D$ such that $A+\nabla \xi(X) \in M^{+}, X \in D$.

Definition 4.2 (Strict quasi-convexity). The function $W$ is strictly quasi-convex at $A \in M^{+}$if and only if equality holds in (4.1) only when $\xi=0$.

Definition 4.3. The function $W \in C\left(M^{+}, \mathbb{R}\right)$ is (strictly) quasi-convex if and only if $W$ is (strictly) quasi-convex at $A$ for all $A \in M^{+}$.

Definition 4.4 (Rank-one convexity). The function $W \in C\left(M^{+}, \mathbb{R}\right)$ is rank-one convex at $A \in M^{+}$if and only if

$$
W(A+\mu a \otimes b) \leq \mu W(A+a \otimes b)+(1-\mu) W(A)
$$

for all $a \in \mathbb{R}, b \in \mathbb{R}$ such that $A+\mu a \otimes b \in M^{+}$where $\mu \in[0,1]$.

Definition 4.5 (Strict rank-one convexity). The function $W \in C\left(M^{+}, \mathbb{R}\right)$ is strictly rank-one convex at $A \in M^{+}$if and only if $W$ is rank-one convex at $A$ and equality in (4.2) holds only when either $a \otimes b=0$ or $\mu(1-\mu)=0$. 
When $W \in C^{1}\left(M^{+}, \mathbb{R}\right)$ is rank-one convex at $A$, the limit $\mu \rightarrow 0$ may be taken in (4.2), which from the definition of a derivative and (2.1) immediately yields the inequality

$$
W(A+a \otimes b) \geq W(A)+\operatorname{tr} T(A)(b \otimes a)
$$

for all $A \in M^{+}, a \in \mathbb{R}^{3}, b \in \mathbb{R}^{3}$ such that $A+a \otimes b \in M^{+}$.

By a suitable translation of coordinates we may take the origin as the interior point with respect to which the bounded region $\Omega$ is supposed star-shaped. Accordingly, we have

$$
N(X) X>0, \quad \forall X \in \partial \Omega,
$$

where as already stated $N(X)$ is the unit outward normal at the point $X$ of $\partial \Omega$.

Let us consider the affine displacement boundary problem whose initial and boundary data (2.3) and (2.4) are affine. That is

$$
u(X)=A X+c+t(B X+d), \quad v(X)=B X+d, \quad X \in \Omega,
$$

and

$$
g(X, t)=A X+c+t(B X+d), \quad(X, t) \in \partial \Omega \times[0,1]
$$

where $A, B$ are constant $3 \times 3$ matrices with positive determinant, and $c, d \in \mathbb{R}^{3}$.

Although solutions for which uniqueness is established are assumed to exist and to be locally smooth, we restrict our attention to those solutions that lie in the class $\mathbb{C}_{1}$ defined explicitly as follows:

Definition 4.6. The smooth functions $f(X, t)$ belong to the class $\mathbb{C}_{1}$ if and only if (a) $W(\nabla f) \in C^{1}\left(M^{+}, \mathbb{R}\right)$, and (b) the following conditions are satisfied:

$$
\begin{gathered}
\sup _{t \in[0,1]} \int_{\Omega(t)}(\nabla f \nabla f+\nabla \dot{f} \nabla \dot{f}) d X \leq M_{1}^{2}, \\
\sup _{t \in[0,1]} \int_{\Omega(t)} \operatorname{tr} T(\nabla f) T^{T}(\nabla f) d X \leq M_{2}^{2},
\end{gathered}
$$

where $M_{1}, M_{2}$ are prescribed constants, and $T(\nabla f)$ is given by the equation corresponding to 2.1).

REMARK 4.7. In the constraint (4.7) the first term on the left may be omitted, and instead an inequality is used that is derived as follows. From the identity

$$
\int_{\Omega(t)} \nabla f \nabla f d X=2 \int_{0}^{t} \int_{\Omega(\eta)} \nabla f \nabla f_{, \eta} d X d \eta+\int_{\Omega(0)} \nabla f \nabla f d X
$$

we employ the arithmetic-geometric mean inequality to obtain

$$
\int_{\Omega(t)} \nabla f \nabla f d X \leq \frac{1}{2} \int_{0}^{t} \int_{\Omega(\eta)} \nabla f \nabla f d X d \eta+2 \int_{0}^{t} \int_{\Omega(\eta)} \nabla f_{, \eta} \nabla f_{, \eta} d X d \eta+\int_{\Omega(0)} \nabla f \nabla f d X .
$$

Integration of the last inequality with respect to $t \in[0,1]$ leads to the bound

$$
\int_{0}^{t} \int_{\Omega(\eta)} \nabla f \nabla f d X d \eta \leq 4 \int_{0}^{t} \int_{\Omega(\eta)} \nabla f_{, \eta} \nabla f_{, \eta} d X d \eta+2 \int_{\Omega(0)} \nabla f \nabla f d X
$$


and then elimination of the first term on the right of (4.9) by means of (4.10) yields

$$
\begin{aligned}
\int_{\Omega(t)} \nabla f \nabla f d X & \leq 4 \int_{0}^{t} \int_{\Omega(\eta)} \nabla f_{, \eta} \nabla f_{, \eta} d X d \eta+2 \int_{\Omega(0)} \nabla f \nabla f d X \\
& \leq 4 M_{1}^{2}+2 \int_{\Omega(0)} \nabla f \nabla f d X,
\end{aligned}
$$

which is the required alternative bound.

We now have

THEOREM 4.8. Let $\Omega$ be star-shaped with respect to an interior point so that (4.4) holds. Let $x(X, t): \bar{\Omega} \times[0,1] \rightarrow \mathbb{R}^{3}$ be a smooth solution corresponding to a strain energy function $W \in C^{2}\left(M^{+}, \mathbb{R}\right)$ that is quasi-convex at $A+t B$ for all $t \in[0,1]$, and strictly rank-one convex. Then in the class $\mathbb{C}_{1}$, the unique solution to the affine initial displacement boundary problem for affine data (4.5) and (4.6) is given by

$$
y(X, t)=A X+c+t(B X+d), \quad(X, t) \in \bar{\Omega} \times[0,1] .
$$

Proof. Assume that the result is false and that $x(X, t) \neq y(X, t)$ is a second solution in the class $\mathbb{C}_{1}$ satisfying the given affine initial and boundary conditions. Introduce the function $H(t)$ defined by (3.1) in terms of $w(X, t)=x(X, t)-y(X, t) \not \equiv 0,(X, t) \in$ $\Omega \times[0,1]$. Then $H(t)$ satisfies the differential inequality (3.9).

By virtue of the assumption that $W$ is quasi-convex at $A+t B$, we have from (2.16) and (4.1) that $G_{1}(t) \geq 0$, and the assumption that $W$ is strictly rank-one convex implies from (2.17) and (4.3) that $G_{2}(t)<0$. Furthermore, it easily follows from (4.12) that $G_{5}(t)$ vanishes identically. Consequently, inequality (3.9) reduces to

$$
H \ddot{H}+(\alpha-1) \dot{H}^{2}<H\left[2 \beta(2 \alpha-1)+2 G_{6}(t)+2 \int_{\Omega(t)} \rho t r \nabla w \dot{w} \otimes X d X\right] .
$$

We separately consider the second and third terms in parenthesis on the right of the last inequality. It follows from (4.12) and (4.8) that (3.11) becomes

$$
\begin{aligned}
G_{6} & =(1+4 \alpha) \int_{0}^{t} \int_{\Omega(\eta)}(t-\eta) \operatorname{tr} B(T(\nabla x)-T(\nabla y)) d X d \eta \\
& \leq Q_{1}
\end{aligned}
$$

where

$$
Q_{1}=(1+4 \alpha)\left[\frac{2}{3} \operatorname{tr} B B^{T} M_{2}^{2}\right]^{\frac{1}{2}} .
$$

With respect to the third term, an integration by parts followed by appeal to Schwarz's inequality, and (3.3) yields

$$
\begin{aligned}
\int_{\Omega(t)} & \rho \operatorname{tr} \nabla w \dot{w} \otimes X d X=-3 \int_{\Omega(t)} \rho w \dot{w} d X-\int_{\Omega(t)} \rho \operatorname{tr} \nabla \dot{w} w \otimes X d X \\
\leq & \left(\int_{\Omega(t)} \rho w w d X\right)^{\frac{1}{2}}\left[3\left(\int_{\Omega(t)} \rho \dot{w} \dot{w} d X\right)^{\frac{1}{2}}+R\left(\int_{\Omega(t)} \rho \nabla \dot{w} \nabla \dot{w} d X\right)^{\frac{1}{2}}\right] \\
< & \dot{H}^{\frac{1}{2}} Q_{2}\left(\lambda, R, M_{1}\right),
\end{aligned}
$$


where the positive constant $Q_{2}$ is given by

$$
Q_{2}=2 \sqrt{2} \rho M_{1}\left[3 \lambda^{-\frac{1}{2}}+R\right],
$$

in which $\lambda$ is the first eigenvalue of the fixed membrane problem on $\Omega$, and

$$
R^{2}=\sup _{\Omega} X X
$$

Consequently, inequality (4.13) becomes

$$
H \ddot{H}+(\alpha-1) \dot{H}^{2}<H\left[2 \dot{H}^{\frac{1}{2}} Q_{2}+2 Q_{1}+2 \beta(2 \alpha-1)\right],
$$

which after an application of the arithmetic-mean geometric mean inequality gives

$$
H \ddot{H}+(\alpha-1) \dot{H}^{2}<\gamma H \dot{H}+H\left[Q_{2}^{2} / \gamma+2 Q_{1}+2 \beta(2 \alpha-1)\right],
$$

where $\gamma$ is an arbitrary positive constant.

For each selected value of the constants $\gamma$ and $\alpha$ we now set

$$
t_{0}^{2}>2(2 \alpha-1), \quad \beta \geq \frac{Q_{2}^{2} / \gamma+2 Q_{1}}{\left[t_{0}^{2}-2(2 \alpha-1)\right]},
$$

so that

$$
\begin{aligned}
Q_{2}^{2} / \gamma+2 Q_{1}+2 \beta(2 \alpha-1) & \leq \beta t_{0}^{2} \\
& \leq \beta\left(t+t_{0}\right)^{2} \\
& <H(t)
\end{aligned}
$$

by (3.1) and the assumed nonidentical vanishing of $w$. Accordingly, from (4.20) we conclude that

$$
H \ddot{H}+(\alpha-1) \dot{H}^{2}<\gamma H \dot{H}+H^{2}, \quad t \in[0,1],
$$

which on setting $z(t)=H^{\alpha}(t)$ may alternatively be written

$$
\ddot{z}<\gamma \dot{z}+\alpha z, \quad t \in[0,1],
$$

or

$$
2[2 \ddot{z}+(-\gamma \pm \delta) \dot{z}]<(\gamma \pm \delta)[2 \dot{z}+(-\gamma \pm \delta) z], \quad t \in[0,1]
$$

where

$$
\delta=\sqrt{\gamma^{2}+4 \alpha} .
$$

Integration of (4.27) and reversion to the function $H(t)$ leads to the following inequality for $t \in[0,1]$ :

$$
\left(\frac{t}{t_{0}}+1\right)^{2}<\exp \frac{\gamma t}{2 \alpha}\left[\cosh \frac{\delta t}{2}\left(1-\frac{\gamma}{\delta} \tanh \frac{\delta t}{2}\right)+\frac{2 \alpha}{\delta t_{0}} \sinh \frac{\delta t}{2}\right]^{\frac{1}{\alpha}}
$$

For each $\alpha$ and $\gamma$ suitable values of $t_{0}$ and $t$ can be selected to obtain a contradiction from (4.29). For example, we may take $\gamma=0.1, \alpha=1.1, t_{0}=1.55$, and $t=0.0155$. We conclude that $w \equiv 0$ on a sufficiently small time interval, and so by iteration $w \equiv 0$ on the whole interval $[0,1]$. This completes the proof of the theorem. 
5. Alternative conditions for uniqueness. Several conditions stipulated in Theorem 4.8 may be relaxed, provided the admissible solutions are further restricted to lie in the class $\mathbb{C}_{2}$ defined by:

Definition 5.1. The smooth functions $f(X, t): \Omega \times[0,1] \rightarrow \mathbb{R}^{3}$ belong to the class $\mathbb{C}_{2}$ if and only they are in the class $\mathbb{C}_{1}$ and the following additional bounds are satisfied:

$$
\begin{array}{r}
\sup _{t \in[0,1]} \int_{\Omega(t)}\left[\operatorname{tr}(\nabla \nabla f)(\nabla \nabla f)^{T}+f_{, \eta \eta} f_{, \eta \eta}+\nabla f_{, \eta \eta} \nabla f_{, \eta \eta}\right] d X \leq M_{3}^{2}, \\
\sup _{t \in[0,1]} \int_{\Omega(t)}|W(\nabla f)| d X \leq M_{4}^{2},
\end{array}
$$

where $M_{3}, M_{4}$ are prescribed constants.

The proof of Theorem4.8 may now be adapted to establish a second uniqueness result.

Theorem 5.2. The initial displacement boundary value problem (2.2)-(2.4) posseses at most one solution in the class $\mathbb{C}_{2}$ subject to the strain energy function $W \in C^{2}\left(M_{+}, \mathbb{R}\right)$ being strictly rank-one convex, and $\Omega$ being star-shaped with respect to an interior point.

Proof. Assume the theorem is false and let $x(X, t): \Omega \times[0,1] \rightarrow \mathbb{R}^{3}$ and $y(X, t):$ $\Omega \times[0,1] \rightarrow \mathbb{R}^{3}$ be two locally smooth distinct solutions to the initial displacement boundary value problem (2.2) (2.4) in the class $\mathbb{C}_{2}$. Set $w(X, t)=x(X, t)-y(X, t) \not \equiv$ $0,(X, t) \in \Omega \times[0,1]$ and consider the function $H(t)$, defined in (3.1), which accordingly satisfies the differential inequality (3.9). The second term on the right of this inequality may be treated as in the previous section and consequently satisfies the bound (4.16), while by virtue of the star-shaped property of $\Omega$ and the strict rank-conexity of $W$ we have $G_{2}(t)<0$. For the remaining terms on the right of (3.9) we appeal to the bounds (4.7), (4.8), (5.1), and (5.2) which by means of operations similar to those used in the proof of Theorem 4.8 leads to

$$
\begin{aligned}
-G_{1}(t) & \leq 2 M_{4}^{2}, \\
G_{5}(t) & \leq \rho M_{1} M_{3}\left[2(4 \alpha+1)+(6+R) \lambda^{-\frac{1}{2}}\right] \\
& \equiv Q_{3}\left(\rho, \alpha, \lambda, R, M_{1}, M_{3}\right), \\
G_{6}(t) & \leq 2 M_{2}\left[2(4 \alpha+1)^{2} M_{1}^{2}+R^{2} M_{3}^{2}\right]^{\frac{1}{2}} \\
& \equiv Q_{4}\left(\alpha, R, M_{2}, M_{3}\right) .
\end{aligned}
$$

Consequently, (3.9) becomes

$$
H \ddot{H}+(\alpha-1) \dot{H}^{2}<H\left[2 \dot{H}^{\frac{1}{2}} Q_{2}+16(\alpha+1) M_{4}^{2}+2 Q_{3}+2 Q_{4}+2 \beta(2 \alpha-1)\right],
$$

which is of the same form as (4.19), and the proof may be completed as before.

REMARK 5.3 (A further restriction). The proof of the previous theorem reveals that the properties of a star-shaped region and of rank-one convexity are nonessential since the arguments remain valid provided only that $G_{2}$ is suitably bounded. This may be achieved by supposing that on $\partial \Omega$ we have $|N X| \leq M_{5}$, a prescribed positive constant, and that the deformation is sufficiently smooth to permit extension of the bounds (4.7), 
(4.8) and (5.2) to $\bar{\Omega} \times[0,1]$. It then follows by Schwarz's inequality that

$$
G_{2}(t) \leq 2 M_{4}^{2} M_{5}^{2}+R M_{1} M_{2} \equiv Q_{5}, \quad t \in[0,1],
$$

so that the right side of (5.6) is modified by the addition of the term $2 Q_{5}$ within the square brackets. The conclusion then follows as previously.

REMARK 5.4. The conclusion of Remark 5.1 indicates that the initial displacement boundary value problem of nonlinear elastodyamics possesses at most one locally smooth solution provided only that certain bounds on the solution are satisfied. Neither a starshaped region nor generalised convexity of the strain energy is required. Such conditions align the result with the corresponding one for linear elastodynamics which requires only existence of a strain-energy function (cf. [19] or [4, [5], and [18) without any sign-definiteness assumptions. The conditions are also similar to those required for the stablisation of an otherwise ill-posed problem (cf. [16]).

Acknowledgement. The author is grateful to Professor C. M. Dafermos for an advanced copy of Chapter V from the second edition of his book 8 .

\section{REFERENCES}

[1] J. M. Ball, Convexity conditions and existence theorems in nonlinear elasticity. Arch. Rational Mechs. Anal. 63 (1976/77), 337-403. MR0475169 (57:14788)

[2] J. M. Ball, Strict convexity, strong ellipticity and regularity in the calculus of variations. Math. Proc. Camb. Phil. Soc. 87 (1980), 501-513. MR0556929 (81d:35014)

[3] J. M. Ball, Some open problems in elasticity. In: Geometry, mechanics, and dynamics: volume in honor of the 60th birthday of J.E.Marsden (ed. by P. Newton, Philip Holmes and Alan Weinstein), Springer-Verlag, New York, 2002, pp 3-59. MR1919825 (2003g:74001)

[4] L. Brun, Sur l'unicité en thermoélasticité dynamique et diverses expressions analogues à la formule de Clapeyron. C. R. Acad. Sci. Paris 261 (1965), 2584-2587. MR0182224 (31:6447)

[5] L. Brun, Méthodes énergétiques dans les systémes évolutifs linéaires. Premiére Partie: Séparation des énergies. Deuxiéme Partie: Théorémes d'unicité. J. de Mech. 8 (1969), 125-166, 167-192.

[6] P. Chadwick, Applications of an energy-momentum tensor in non-linear elasticity. J. Elasticity 5 (1975), 249-258. MR0462093 (57:2068)

[7] C. M. Dafermos, The second law of thermodynamics and stability. Arch. Rational Mech. Anal. 70 (1979), 167-179. MR0546634 (80j:73004)

[8] C. M. Dafermos, Hyperbolic Conservation Laws in Continuum Physics. Second Edition. SpringerVerlag, Berlin, 2005. MR2169977 (2006d:35159)

[9] J. L. Ericksen, Special topics in elastostatics. In: Yih, C-S (ed.) Advances in Applied Mechanics, Academic Press, New York, 1977, pp. 189-244.

[10] J. D. Eshelby, The elastic energy-momentum tensor. J. Elasticity 5 (1975), 321-335. MR0489190 $(58: 8645)$

[11] D. C. Fletcher, Conservation laws in linear elastodynamics. Arch. Rational Mech. Anal. 60 (1976), 329-353. MR0421261 (54:9266)

[12] I. M. Gelfand and S. V. Fomin, Calculus of Variations. Prentice-Hall, Englewood Cliffs, NJ, 1963. MR0160139(28:3353)

[13] A. E. Green, On some general formulae in finite elastostatics. Arch. Rational Mech. Anal. 50 (1973), 73-80. MR0441065 (55:13931)

[14] R. Hill, Energy-momentum tensors in elastostatics: some reflections on the general theory. J. Mech. Phys. Solids 34 (1986), 305-317. MR0844951 (87f:73017)

[15] T. J. R. Hughes, T. Kato and J. E. Marsden, Well-posed quasi-linear second-order hyberbolic systems with applications to nonlinear elastodynamics and general relativity. Arch. Rational Mech. Anal. 63 (1976), 273-294. MR0420024 (54:8041)

[16] F. John, Continuous dependence on data for solutions of partial differential equations with a prescribed bound. Comm. Pure Appl. Mathematics 13 (1960), 551-585. MR0130456 (24:A317) 
[17] R. J. Knops, On uniqueness in nonlinear homogeneous elasticity. In: Rational Continua Classical and New (ed. by P. Podio-Guidugli and M. Brocato) Springer, Milano, 2003, pp. 119-137. MR 2014184 (2004k:74020)

[18] R. J. Knops and L. E. Payne, Uniqueness in classical elasticity. Arch. Rational Mech. Anal. 27 (1967), 349-355. MR0219261 (36:2344)

[19] R. J. Knops and L. E. Payne, Uniqueness Theorems in Linear Elasticity. Springer Tracts in Natural Philosophy, vol. 19, Springer-Verlag, Berlin, 1971. MR0421244 (54:9249)

[20] R. J. Knops, H. A. Levine and L. E. Payne, Non-existence, instability, and growth theorems for solutions of a class of abstract nonlinear equations with applications to nonlinear elastodynamics. Arch. Rational Mech. Anal. 55 (1974), 52-72. MR0364839 (51:1093)

[21] R. J. Knops and C. A. Stuart, Quasiconvexity and uniqueness of equilibrium solutions in nonlinear elasticity. Arch. Rational Mech. Anal. 86 (1984), 233-249. MR0751508 (85j:73012)

[22] J. K. Knowles and E. Sternberg, On a class of conservation laws in linearized and finite elastostatics. Arch. Rational Mech. Anal. 44 (1972), 187-211. MR0337111(49:1883)

[23] G. Maugin, Material Inhomogeneities in Elasticity. Chapman and Hall, London, 1993. MR1250832 (95c:73001)

[24] S. Müller, Variational models for microstructure and phase transitions. In: Proc. C.I.M.E. Summer School "Calculus of Variations and Geometric Evolution Problems" (ed. by S. Hildebrandt and M. Struwe), Cetraro. Lecture Notes in Mathematics, vol. 1713, Springer-Verlag, Berlin, 1996, pp. 85-210. MR1731640 (2001b:49019)

[25] M. Sofer, A note on the growth of trajectories in nonlinear elastodynamics. J. Elasticity 28 (1992), 185-192. MR 1173356 (93g:73050)

[26] L. Wheeler, A uniqueness theorem for the displacement problem in finite elastodynamics. Arch. Rational Mech. Anal. 63 (1977), 183-189. MR0421253 (54:9258) 